\title{
25
}

\section{On the Optimal Timing of Software Development Cycle}

\author{
Hakman A. Wan \\ Department of Computer Studies, Lingnan College, Hong Kong
}

\begin{abstract}
This paper puts forth a mathematical model and studies the optimal time to start the development cycle of a system before the existing system retires. By assuming a log-linear cost function of maintenance expenses, the solution to the optimization problem can be found.
\end{abstract}

Keyword Codes: K.6.1; K.6.4

Keywords: Replacement problem, optimization, Lagrangean multiplier

\section{INTRODUCTION}

Dissatisfactions against an old system pile up until management decides to initiate another round of the system development cycle. But until the new system becomes operational, the existing one remains the only system available to the users. The inappropriateness [1] of the old system still require maintenance which incurs substantial manpower and cost. An early start of the new cycle obviously reduces spending on old system maintenance. But it shortens the operational lifespan of the old system and hence discourages the management to spend time in system development stages. To determine the optimal time for a start to re-write a new system is known as the Software Replacement Problem.

The Software Replacement Problem is studied by a handful of researchers among them are Gode et al. [2] and Chung [3]. The latter devises a cost function which totals the maintenance and re-writing cost over the planning horizon. A hypothetically perfect productivity of the development team is shown to incur instantaneous replacement. However, we redesign the cost function and produce a solution which differs from instantaneous replacement.

\section{THE MODEL}

We consider two consecutive system development cycles. Let $T_{R}$ be the time when the new system cycle begins (ie. re-writing the system starts) and $T_{N}$ be the time when the new system begins operational. The expenditure during this period includes: 1) maintenance cost between time 0 to $T_{N}, 2$ ) development cost between time $T_{R}$ to $T_{N}$, and 3) maintenance cost between time $T_{N}$ to $T$. Our problem is to find the timing of $T_{R}$ such that the sum of the first two costs is minimized.

Let $m(0)$ represent the number of maintenance requests at time 0 , when the old system begins to function. Hence the number of maintenance requests at time $t$ can be expressed as

$$
m(t)=m(0) e^{\delta t}
$$

where $\delta>0$ is the logarithmic rate of growth which can be determined by regression analysis. Assuming that every request is entertained by the maintenance team, $m(t)$ becomes the number of requests serviced. It is believed that $\delta$ reflects the willingness of the maintenance 
team to respond to these requests. If the attitude of the team does not change, $m(t)$ is a monotonically increasing function. However, some change of $\delta$ is expected and it brings about different considerations which will be mentioned later.

Let $h: R \rightarrow R$ be the cost function which maps number of maintenance requests to monetary units of expenses. Thus the total maintenance expenses during the period $(0, t)$ is

$$
\int_{0}^{t} h(m(t)) d t
$$

We employ a log-linear cost function $h$. Then the (present value of the) cost function can be expressed as

$$
h(m(t))=\alpha[m(t)]^{\beta} e^{-r t}
$$

where $\alpha$ and $\beta$ are defined respectively by the structuredness of the programming technique on the system development and the productivity of the system developers; and $r>0$ is the rate of interest. The total maintenance cost in the period $\left(0, T_{R}\right)$ is

$$
\int_{0}^{T_{R}} \alpha[m(t)]^{\beta} e^{-r t} d t=\frac{\alpha m^{\beta}(0)}{\delta \beta-r}\left(e^{(\delta \beta-r) T_{R}}-1\right)
$$

The maintenance cost in the period $\left(T_{R}, T_{N}\right)$ is not included in the integral because we suspect that the system developers may have a different attitude towards maintenance when they learn that the system will soon be replaced. The number of maintenance requests may grow at the same rate $\delta$, but the system team will then be reluctant to entertain as many of these requests as they did before $T_{R}$. Here we assume a different rate of growth (ie, we let $\delta_{1}$ replace $\delta$ and let $\delta_{2}$ represent the rate of growth of maintenance requests that will be serviced. N.B. $\delta_{1}>\delta_{2}$.) Thus expressions (1) and (4) should be

$$
m(t)=m(0) e^{\delta_{1} t} \quad \text { and } \quad \frac{\alpha m^{\beta}(0)}{\delta_{1} \beta-\tau}\left(e^{\left(\delta_{1} \beta-r\right) T_{R}}-1\right)
$$

The last one corresponds to the total cost of maintenance in the period $\left(0, T_{R}\right)$. Following the same argument, the total maintenance cost in the period $\left(T_{R}, T_{N}\right)$ is found to be

$$
\frac{\alpha m^{\beta}(0) e^{\beta T_{R}}}{\delta_{2} \beta-r}\left(e^{\left(\delta_{2} \beta-r\right) T_{N}}-e^{\left(\delta_{2} \beta-r\right) T_{R}}\right)
$$

On the other hand, the rewriting effort is determined by $\int_{T_{N}}^{T_{R}} L d t$ where $L$ represents the daily wages paid to the project team. Hence the total effort, if expressed in terms of its present value, is

$$
\frac{\alpha m^{\beta}(0)}{\delta_{1} \beta-r}\left(e^{\left(\delta_{1} \beta-r\right) T_{R}}-1\right)+\frac{\alpha m^{\beta}(0) e^{\delta_{1} \beta T_{R}}}{\delta_{2} \beta-r}\left(e^{\left(\delta_{2} \beta-r\right) T_{N}}-e^{\left(\delta_{2} \beta-r\right) T_{R}}\right)-\frac{L}{r}\left[e^{-r T_{R}}-e^{-r T_{N}}\right]
$$

In reality, the effort of developing a system is related to the complexity of the system where complexity can be approximated by the number of function points $[3,4]$. Thus $L=\frac{F}{\mu}$ where $F$ is the measure of function points and $\mu$ is a proportional constant.

\section{THE PROBLEM AND THE SOLUTION}

Thus we have an optimization problem:

To minimize $\quad E\left(T_{R}, T_{N}\right) \equiv$

$$
\frac{\alpha m^{\beta}(0)}{\delta_{1} \beta-r}\left(e^{\left(\delta_{1} \beta-r\right) T_{R}}-1\right)+\frac{\alpha m^{\beta}(0) e^{\delta_{1} \beta T_{R}}}{\delta_{2} \beta-r}\left(e^{\left(\delta_{2} \beta-r\right) T_{N}}-e^{\left(\delta_{2} \beta-r\right) T_{R}}\right)-\frac{F}{\mu r}\left[e^{-r T_{R}}-e^{-r T_{N}}\right]
$$


such that $T_{N} \leq T_{R}$.

Let $\gamma$ be the Lagrangean multiplier and

$$
f\left(E\left(T_{R}, T_{N}\right), \gamma\right)=E\left(T_{R}, T_{N}\right)+\gamma\left(T_{R}-T_{N}+s^{2}\right)
$$

be the Lagrangean function. Since $E\left(T_{R}, T_{N}\right)$ is convex, the Kuhn-Tucker conditions for minimization are

$$
\begin{aligned}
& \alpha m^{\beta}(0)\left[e^{\left(\delta_{1} \beta-4\right) T_{R}}-e^{\left(\delta_{1} \beta+\delta_{2} \beta-\tau\right) T_{R}}\right]+ \\
& \frac{\alpha m^{\beta}(0) \delta_{1} \beta e^{\delta_{1} \beta T_{R}}}{\delta_{2} \beta-r}\left[e^{\left(\delta_{2} \beta-r\right) T_{N}}-e^{\left(\delta_{2} \beta-r\right) T_{R}}\right]+\frac{F}{\mu} e^{-r T_{R}}+\gamma=0 \\
& \alpha m^{\beta}(0) e^{\delta_{1} \beta T_{R}} e\left(\delta_{2} \beta-r\right) T_{N}-\frac{F}{\mu} e^{-r T_{N}}+\gamma=0 \\
& T_{R}-T_{N}+s^{2}=0 \\
& \gamma s=0 \\
& \gamma \geq 0
\end{aligned}
$$

Solving equations (9)-(13) we look into two different cases:

Case 1 If $s=0$, then from (11) $T_{N}=T_{R}$. It is the so-called instantaneous replacement solution which suggests an non-existent development period for the new system. Assume that $T=T_{N}=T_{R}$. Equations (9)-(13) give rise to

$$
\frac{2 L e^{-\delta_{1} \beta T}}{\alpha m^{\beta}(0)}=2 e^{\delta_{2} \beta T}-1
$$

We can then derive the value of $T$ if all the other variables are known. If we assume further that $\delta=\delta_{1}=\delta_{2}$, (14) can be simplified to

$$
T=\frac{1}{\delta \beta} \ln \left(\frac{1+\sqrt{1+8 A}}{4}\right)
$$

where $A=\frac{2 L}{\delta m^{\beta}(0)}$. Theoretically speaking, $T_{N}=T_{R}=T$ is possible if the values are very large numbers (ie. the new system will never begin its development.) It can be accomplished if $\beta$ is extremely small (ie, the productivity of the development team is extremely high), $\alpha$ is extremely small (ie, the existing system has always been developed in a highly structured way). However, since $T>0, A$ or $L$ must be sufficiently large; ie

$$
L>\frac{1}{2} \alpha m^{\beta}(0)
$$

Case 2 If $\gamma=0$ then $s^{2}>0$ or $T_{N}>T R$. The relation between $T_{N}$ and $T_{R}$ is given by (10)

$$
\frac{T_{R}}{T_{N}}=\frac{1}{\delta_{1} \beta T_{N}} \ln \left(\frac{A}{2}\right)-d
$$

where $d=\delta_{2} / \delta_{1}$. The optimal timing to start developing the new system can be found by solving (17). However, since $0<T_{R}<T_{N}$

$$
1>\frac{T_{R}}{T_{N}}=\frac{1}{\delta_{1} \beta T_{N}} \ln \left(\frac{A}{2}\right)-d>0
$$


The inequalities can be simplified to

$$
h\left(m\left(T_{N}\right)\right) e^{\delta_{2} \beta T_{N}}>L>\alpha m^{\beta}(0) e^{\delta_{2} \beta T_{N}}
$$

In general, $1>d>0$. The right side of (18) ranges from $h\left(m\left(T_{N}\right)\right)$ and $\alpha m^{\beta}(0)$. When $d=1$, the inequality gives

$$
L>h\left(m\left(T_{N}\right)\right)
$$

It implies that if all maintenance requests are serviced as if no new system is being developed, the cost of developing a new system is still higher than the maintenance cost at the time when the new system is operational. But if $d=0$, ie, all maintenance requests are ignored after the new development cycle commences, the left side of $(18)$ gives $h\left(m\left(T_{N}\right)\right)>L$. ie, the expenses of developing the new system must be smaller than the maintenance cost at $T_{N}$, should there be any.

Again, from (17), the structuredness $\alpha$ of the existing system has a positive effect on the ratio $T_{R} / T_{N}$ since a small $\alpha$ (ie, a highly structured old system) prolongs the lifespan of the system by increasing the ratio $T_{R} / T_{N}$.

\section{CONCLUSION}

Several ideas can be derived from the solutions of the problem:

1. It is discovered that $r$, the rate of interest, does not appear in any of the solutions. It can be certain that it casts no influence on the value of $T_{R}$.

2. If we put the instantaneous replacement strategy to practice, we must expand the lifespan of the existing system as long as possible. It demands a maintenance team of high productivity and an extremely structured development technology, as analyzed in case 1 .

3. In both cases, the value of $L$ is bounded by a value proportional to $\alpha m^{\beta}(0)$. From (17), we learn that a highly structured system should last longer than a less structured one, but the influence of $\alpha$ is not very conspicuous since it is hidden in a logarithmic function.

4. The policy of providing selective responses to all the maintenance requests after a new development cycle begins has a positive effect on prolonging the lifespan of the existing system. From (18), the ratio $T_{R} / T_{N}$ is increased if a small $d$ is chosen.

\section{References}

1 Davis, A.M., Bersoff, E.H., and Comer, E.R., A Strategy for Comparing Alternative Software Development Life Cycle Models, IEEE Trans. Software Engineering, vol 14. No. 10 , Oct 1988.

2 Gode, D.K, Barua, A., and Mukhopadhyay, T., On the Economics of the Software Replacement Problem, Proceedings of the 11th International Conference on Information Systems, 1990.

3 Chung, S.L., Timing of Software Replacement, preprint.

4 Albrecht, A.J. and Gaffney, J.E., Software Function, Source Lines of Code, and Development Effort Prediction: A Software Science Validation, IEEE Trans. Soft. Eng., vol SE-9, No. 6, Nov 1983. 